PROCEEDINGS OF THE

AMERICAN MATHEMATICAL SOCIETY

Volume 134, Number 4, Pages 1197-1204

S 0002-9939(05)08045-7

Article electronically published on July 20, 2005

\title{
EMBEDDED MINIMAL DISKS WITH PRESCRIBED CURVATURE BLOWUP
}

\author{
BRIAN DEAN
}

(Communicated by Richard A. Wentworth)

\begin{abstract}
We construct a sequence of compact embedded minimal disks in a ball in $\mathbb{R}^{3}$, whose boundaries lie in the boundary of the ball, such that the curvature blows up only at a prescribed discrete (and hence, finite) set of points on the $x_{3}$-axis. This extends a result of Colding and Minicozzi, who constructed a sequence for which the curvature blows up only at the center of the ball, and is a partial affirmative answer to the larger question of the existence of a sequence for which the curvature blows up precisely on a prescribed closed set on the $x_{3}$-axis.
\end{abstract}

In 1, T.H. Colding and W.P. Minicozzi II constructed a sequence of compact embedded minimal disks in a ball in $\mathbb{R}^{3}$, with boundaries lying in the boundary of the ball, such that the curvature blows up only at the center. This result raises the following question.

Question 1. Does there exist a sequence of compact embedded minimal disks in a ball in $\mathbb{R}^{3}$, whose boundaries lie in the boundary of the ball, such that the curvature blows up precisely on a prescribed closed set on the $x_{3}$ - axis?

Beyond that, it is interesting to consider which curves can arise as the singular set for curvature of a sequence of embedded minimal disks. W. Meeks and M. Weber have constructed examples (see 3]) in which the singular set is a circle.

By scaling, it suffices to consider embedded minimal disks in the unit ball. Our main result says that the answer to Question 1 is affirmative in the case where the closed set is a discrete (and hence, finite) set of points.

Theorem 2. Given $n$ points $\left(0,0, b_{j}\right) \subset B_{1}, b_{1}<\ldots<b_{n}$, there is a sequence of compact embedded minimal disks $0 \in \Sigma_{i} \subset B_{1} \subset \mathbb{R}^{3}$ with $\partial \Sigma_{i} \subset \partial B_{1}$ and containing the vertical segment $\{(0,0, t):|t|<1\} \subset \Sigma_{i}$, and such that the following hold:

(i) $\lim _{i \rightarrow \infty}\left|A_{\Sigma_{i}}\right|^{2}\left(0,0, b_{j}\right)=\infty, j=1, \ldots, n$.

(ii) $\sup _{i} \sup _{\Sigma_{i} \backslash \bigcup_{j} B_{\delta_{j}}\left(0,0, b_{j}\right)}\left|A_{\Sigma_{i}}\right|^{2}<\infty$ for all $\delta_{j}>0, j=1, \ldots, n$.

(iii) $\Sigma_{i} \backslash\left\{x_{3}\right.$-axis $\}=\Sigma_{1, i} \cup \Sigma_{2, i}$ for multi-valued graphs $\Sigma_{1, i}$ and $\Sigma_{2, i}$.

(iv) $\Sigma_{i} \backslash \bigcup_{j}\left\{x_{3}=b_{j}\right\}$ converges to $n+1$ embedded minimal disks $\Sigma^{k}, k=$ $1, \ldots, n+1$, satisfying the following:

(a) $\Sigma^{k} \subset\left\{x_{3}<b_{k}\right\}$ for $k=1, \ldots, n$, and $\Sigma^{n+1} \subset\left\{x_{3}>b_{n}\right\}$.

Received by the editors August 10, 2004 and, in revised form, October 26, 2004.

2000 Mathematics Subject Classification. Primary 53C42; Secondary 53A10, 57R40.

The author thanks W. Minicozzi for his many helpful discussions.

(C)2005 American Mathematical Society Reverts to public domain 28 years from publication 
(b) $\overline{\Sigma^{1}} \backslash \Sigma^{1}=B_{1} \cap\left\{x_{3}=b_{1}\right\}, \overline{\Sigma^{n+1}} \backslash \Sigma^{n+1}=B_{1} \cap\left\{x_{3}=b_{n}\right\}$, and for $k=2, \ldots, n, \overline{\Sigma^{k}} \backslash \Sigma^{k}=B_{1} \cap\left(\left\{x_{3}=b_{k-1}\right\} \cup\left\{x_{3}=b_{k}\right\}\right)$.

(c) $\Sigma^{1} \backslash\left\{x_{3}-\right.$ axis $\}=\Sigma_{1}^{1} \cup \Sigma_{2}^{1}$ for multi-valued graphs $\Sigma_{1}^{1}$ and $\Sigma_{2}^{1}$ each of which spirals into $\left\{x_{3}=b_{1}\right\} . \Sigma^{n+1} \backslash\left\{x_{3}-\right.$ axis $\}=\Sigma_{1}^{n+1} \cup \Sigma_{2}^{n+1}$ for multi-valued graphs $\Sigma_{1}^{n+1}$ and $\Sigma_{2}^{n+1}$ each of which spirals into $\left\{x_{3}=\right.$ $\left.b_{n}\right\}$. For $k=2, \ldots, n, \Sigma^{k} \backslash\left\{x_{3}-\right.$ axis $\}=\Sigma_{1}^{k} \cup \Sigma_{2}^{k}$ for multi-valued graphs $\Sigma_{1}^{k}$ and $\Sigma_{2}^{k}$ each of which spirals into $\left\{x_{3}=b_{k-1}\right\}$ and $\left\{x_{3}=\right.$ $\left.b_{k}\right\}$.

Question 1 remains open for closed sets in general; for example, closed intervals or Cantor-type sets. We conjecture that the answer is affirmative in general. Let us briefly discuss how one might show this. One idea is to note that, given any closed set, there exists a countable dense subset. One would want to construct a sequence of compact embedded minimal disks whose curvature blows up on the countable dense subset. By Theorem 2, such a sequence exists for any set of $n$ points, for any fixed finite $n$; one would then want to let $n \rightarrow \infty$ and use a diagonal argument to obtain a sequence whose curvature blows up on the countable set of points in the dense subset. As a result of [2, Lemma I.1.4], the set of points on which the curvature blows up must be closed. Hence, the curvature would blow up on the closure of the countable dense subset, which is precisely our prescribed closed set.

The key to extending Theorem 2 from finitely many to countably many points would be to show that all of the intermediate results we use in this paper to prove Theorem 2 hold uniformly in $n$. As we prove these intermediate results, most of them will be easily seen to hold uniformly in $n$. However, it is not clear whether or not part (iii) in Lemma 5 is uniform; it appears that the number $r_{0}$ which we obtain depends on $n$, and approaches 0 as $n$ tends to infinity.

We now return to the issue at hand: the finitely many points case. Theorem 2 says the following. Given $n$ points on the $x_{3}$-axis, $\left(0,0, b_{j}\right)$ for $j=1, \ldots, n$, with $b_{1}<\ldots<b_{n}$, we construct a sequence of disks $\Sigma_{i} \subset B_{1}=B_{1}(0) \subset \mathbb{R}^{3}$ where the curvatures blow up only at the prescribed $n$ points, and $\Sigma_{i} \backslash\left\{x_{3}\right.$-axis $\}$ consists of two multi-valued graphs for each $i$. The sequence $\Sigma_{i} \backslash \bigcup_{j}\left\{x_{3}=b_{j}\right\}$ converges to $n+1$ embedded minimal disks $\Sigma^{k}$, which sit between and spiral into the appropriate planes $\left\{x_{3}=b_{j}\right\}$. The result of Colding and Minicozzi in [1] is just Theorem 2 with $n=1$ and $b_{1}=0$.

For the reader's convenience, we will structure this paper similar to [1. In particular, we provide some of the brief background on the Weierstrass representation which Colding and Minicozzi also outlined.

Let $\Omega \subset \mathbb{C}$ be a domain. The Weierstrass representation is as follows (see, for example, 4]). Given any meromorphic function $g$ on $\Omega$ and any holomorphic oneform $\phi$ on $\Omega$, we obtain a (branched) conformal minimal immersion $F: \Omega \rightarrow \mathbb{R}^{3}$, where

$$
F(z)=\operatorname{Re} \int_{\zeta \in \gamma_{z_{0}, z}}\left(\frac{1}{2}\left(g^{-1}(\zeta)-g(\zeta)\right), \frac{i}{2}\left(g^{-1}(\zeta)+g(\zeta)\right), 1\right) \phi(\zeta) .
$$

Here, we are integrating along a path $\gamma_{z_{0}, z}$ from a fixed base point $z_{0}$ to $z$. The choice of $z_{0}$ changes $F$ by adding a constant. We will assume that $F(z)$ is independent of the choice of path, which is the case, for example, when $g$ has no zeros or poles and $\Omega$ is simply connected (and this will be the case for our choices of $g$ and $\Omega$. 
The unit normal $\mathbf{n}$ and Gauss curvature $K$ of the resulting minimal surface are given by (see [4, Sec. 8,9])

$$
\begin{gathered}
\mathbf{n}=\left(2 \operatorname{Re} g, 2 \operatorname{Im} g,|g|^{2}-1\right) /\left(|g|^{2}+1\right), \\
K=-\left[\frac{4\left|\partial_{z} g\right||g|}{|\phi|\left(1+|g|^{2}\right)^{2}}\right]^{2}
\end{gathered}
$$

The one-form $\phi$ is called the height differential, and by equation (2), $g$ is the composition of the Gauss map followed by stereographic projection.

We will assume that $\phi$ does not vanish and $g$ has no zeros or poles; this implies that $F$ is an immersion, i.e., $d F \neq 0$. One of the standard examples of this, which has the added benefit of being an $\infty$-valued graph, and hence interesting for our purposes, is the helicoid, whose Weierstrass data are

$$
g(z)=e^{i z}, \phi(z)=d z, \Omega=\mathbb{C} .
$$

This motivates the following. If we want to construct multi-valued minimal graphs, perhaps we should consider Weierstrass data of the form

$$
g(z)=e^{i h(z)}=e^{i(u(z)+i v(z))}, \phi(z)=d z,
$$

for an appropriate choice of $\Omega$, where $h(z)$ is a holomorphic function. The next lemma gives us the differential of $F$ in this case.

Lemma 3. If $F$ is given by equation (11) with $g(z)=e^{i(u(z)+i v(z))}$ and $\phi(z)=d z$, then

$$
\begin{gathered}
\partial_{x} F=(\sinh v \cos u, \sinh v \sin u, 1), \\
\partial_{y} F=(\cosh v \sin u,-\cosh v \cos u, 0) .
\end{gathered}
$$

In particular, for the proof of Theorem 2 we will construct our multi-valued minimal graphs in this way, with our choices of function $h_{a}(z)$ and domain $\Omega_{a}$ varying for each element of the sequence. That is, we will construct a one-parameter family of minimal immersions $F_{a}, a \in(0,1 / 2)$, with Weierstrass data $g=e^{i h_{a}}$ (where $h_{a}=u_{a}+i v_{a}$ ), $\phi=d z$, and domains $\Omega_{a}$ which we will specify shortly. We will prove that this family of immersions is compact in Lemma 4, and that the immersions $F_{a}: \Omega_{a} \rightarrow \mathbb{R}^{3}$ are embeddings in Lemma 5 .

For each $0<a<1 / 2$, let

$$
\begin{aligned}
h_{a}(z)= & \sum_{j=1}^{n} \frac{1}{2^{j-1} a} \arctan \left(\frac{z-b_{j}}{a}\right) \text { on } \Omega_{a}=\bigcup_{j=1}^{n} \Omega_{a, j}, \text { where } \\
\Omega_{a, 1}= & \left\{(x, y):-\frac{1}{2} \leq x \leq \frac{b_{2}-b_{1}}{2},|y| \leq \frac{\left[\left(x-b_{1}\right)^{2}+a^{2}\right]^{3 / 4}}{2}\right\}, \\
\Omega_{a, j}= & \left\{(x, y): \frac{b_{j}-b_{j-1}}{2} \leq x \leq \frac{b_{j+1}-b_{j}}{2},|y| \leq \frac{\left[\left(x-b_{j}\right)^{2}+a^{2}\right]^{3 / 4}}{2}\right\}, \\
& j=2, \ldots, n-1, \\
\Omega_{a, n}= & \left\{(x, y): \frac{b_{n}-b_{n-1}}{2} \leq x \leq \frac{1}{2},|y| \leq \frac{\left[\left(x-b_{n}\right)^{2}+a^{2}\right]^{3 / 4}}{2}\right\} .
\end{aligned}
$$

To get an idea of what $\Omega_{a}$ looks like, note that the $\Omega_{a, j}$ are defined similar to the domain called $\Omega_{a}$ by Colding and Minicozzi (see [1, Figure 4]), only centered at $b_{j}$ 
instead of at 0 . When $a \rightarrow 0$, the domain pinches off at the $n$ points $b_{j}$, just as Colding and Minicozzi's domain pinches off at 0 (see [1, Figure 5]).

Note that $h_{a}$ is well defined, since $\Omega_{a}$ is simply connected and $b_{j} \pm i a \notin \Omega_{a}$ for $j=1, \ldots, n$. By direct computation, we see that

$$
\begin{aligned}
\partial_{z} h_{a}(z) & =\sum_{j=1}^{n} \frac{1}{2^{j-1}} \frac{1}{\left(z-b_{j}\right)^{2}+a^{2}} \\
& =\sum_{j=1}^{n} \frac{1}{2^{j-1}} \frac{\left(x-b_{j}\right)^{2}+a^{2}-y^{2}-2 i\left(x-b_{j}\right) y}{\left[\left(x-b_{j}\right)^{2}+a^{2}-y^{2}\right]^{2}+4\left(x-b_{j}\right)^{2} y^{2}} .
\end{aligned}
$$

By the Cauchy-Riemann equations, we get

$$
\partial_{z} h_{a}=\partial_{x} u_{a}-i \partial_{y} u_{a}=\partial_{y} v_{a}+i \partial_{x} v_{a} .
$$

Also, the curvature is given by (see equation (30)

$$
\begin{aligned}
K_{a}(z) & =\frac{-\left|\partial_{z} h_{a}\right|^{2}}{\cosh ^{4} v_{a}} \\
& =-\frac{\left|\sum_{j=1}^{n} 2^{1-j}\left(\left(z-b_{j}\right)^{2}+a^{2}\right)^{-1}\right|^{2}}{\cosh ^{4}\left(\operatorname{Im}\left(\sum_{j=1}^{n} \arctan \left(\left(z-b_{j}\right) / a\right) / 2^{j-1} a\right)\right)} .
\end{aligned}
$$

Note that $\lim _{a \rightarrow 0}\left|K_{a}(z)\right|=\infty$ for $z=b_{j}, j=1, \ldots, n$.

Let $F_{a}: \Omega_{a} \rightarrow \mathbb{R}^{3}$ be from equation (1) with $g=e^{i h_{a}}, \phi=d z$, and $z_{0}=0$. Let $\Omega_{0}=\bigcap_{a} \Omega_{a} \backslash\left\{b_{1}, \ldots, b_{n}\right\}$. The family of functions $h_{a}$ is not compact, since $\lim _{a \rightarrow 0}\left|h_{a}\right|(z)=\infty$ for $z \in \Omega_{0}$. However, as the following lemma shows, the family of immersions $F_{a}$ is compact.

Lemma 4. If $a_{k} \rightarrow 0$, there exists a subsequence, which we also call $a_{k}$, such that $F_{a_{k}}$ converges uniformly in $C^{2}$ on compact subsets of $\Omega_{0}$.

Proof. Similar to the proof of [1, Lemma 2], with

$$
-\sum_{j=1}^{n} \frac{1}{2^{j-1}} \frac{1}{z-b_{j}}
$$

in place of $-1 / z$.

In the next lemma, we show that the immersions $F_{a}: \Omega_{a} \rightarrow \mathbb{R}^{3}$ are in fact embeddings. This will follow from parts (i) and (ii) of the lemma. Part (i) says that the slice $\left\{x_{3}=t\right\} \cap F_{a}\left(\Omega_{a}\right)$ is the image of the segment $\{x=t\}$ in the plane; that is, as $x$ varies and $y$ stays fixed, there is no self-intersection. In part (ii), we show that, in each slice $\left\{x_{3}=t\right\} \cap F_{a}\left(\Omega_{a}\right)$, the image $F_{a}\left(\{x=t\} \cap \Omega_{a}\right)$ is a graph over some line segment in the slice; that is, as $y$ varies and $x$ stays fixed, there is no self-intersection.

Lemma 5. For all $a>0$, the immersions $F_{a}: \Omega_{a} \rightarrow \mathbb{R}^{3}$ satisfy

(i) $x_{3}\left(F_{a}(x, y)\right)=x$.

(ii) For each fixed $x, F_{a}(x, \cdot)$ is a graph in the plane $\left\{x_{3}=x\right\}$.

(iii) There exists $r_{0}>0$ such that, for all $a$,

$$
\left|F_{a}\left(x, \pm \frac{\left[\left(x-b_{1}\right)^{2}+a^{2}\right]^{3 / 4}}{2}\right)-F_{a}(x, 0)\right|>r_{0},-\frac{1}{2} \leq x \leq \frac{b_{2}-b_{1}}{2}
$$




$$
\begin{aligned}
\left|F_{a}\left(x, \pm \frac{\left[\left(x-b_{j}\right)^{2}+a^{2}\right]^{3 / 4}}{2}\right)-F_{a}(x, 0)\right|> & r_{0}, \frac{b_{j}-b_{j-1}}{2} \leq x \leq \frac{b_{j+1}-b_{j}}{2}, \\
& j=2, \ldots, n-1, \\
\left|F_{a}\left(x, \pm \frac{\left[\left(x-b_{n}\right)^{2}+a^{2}\right]^{3 / 4}}{2}\right)-F_{a}(x, 0)\right|> & r_{0}, \frac{b_{n}-b_{n-1}}{2} \leq x \leq \frac{1}{2} .
\end{aligned}
$$

Proof. (i) is immediate by the definition of $F_{a}$, since $z_{0}=0$ and $\phi=d z$.

To prove (ii), first note that, by equations (8) and (9), we have

$$
\left|\partial_{y} u_{a}(x, y)\right| \leq \sum_{j=1}^{n} \frac{1}{2^{j-1}} \frac{2\left|x-b_{j}\right||y|}{\left[\left(x-b_{j}\right)^{2}+a^{2}-y^{2}\right]^{2}+4\left(x-b_{j}\right)^{2} y^{2}} .
$$

Fix $k, 1 \leq k \leq n$. On $\Omega_{a, k}$ (where $\left.\left(x-b_{k}\right)^{2}=\min _{j}\left(x-b_{j}\right)^{2}\right)$, we have, for all $j=1, \ldots, n$,

$$
\begin{aligned}
{\left[\left(x-b_{j}\right)^{2}+a^{2}-y^{2}\right]^{2}+4\left(x-b_{j}\right)^{2} y^{2} } & \geq\left[\left(x-b_{j}\right)^{2}+a^{2}-y^{2}\right]^{2} \\
& \geq\left[\left(x-b_{j}\right)^{2}+a^{2}-\frac{\left(x-b_{k}\right)^{2}+a^{2}}{4}\right]^{2} \\
& \geq\left[\left(x-b_{j}\right)^{2}+a^{2}-\frac{\left(x-b_{j}\right)^{2}+a^{2}}{4}\right]^{2} \\
& =\frac{9}{16}\left[\left(x-b_{j}\right)^{2}+a^{2}\right]^{2} .
\end{aligned}
$$

Therefore, we have

$$
\left|\partial_{y} u_{a}(x, y)\right| \leq 4 \sum_{j=1}^{n} \frac{1}{2^{j-1}} \frac{\left|x-b_{j}\right||y|}{\left[\left(x-b_{j}\right)^{2}+a^{2}\right]^{2}} .
$$

Set $y_{x, a, k}=\frac{\left[\left(x-b_{k}\right)^{2}+a^{2}\right]^{3 / 4}}{2}$. Integrating (11) gives

$$
\begin{aligned}
\max _{|y| \leq y_{x, a, k}}\left|u_{a}(x, y)-u_{a}(x, 0)\right| & \leq \max _{|y| \leq y_{x, a, k}}\left|\int_{0}^{y} \partial_{y} u_{a}(x, t) d t\right| \\
& \leq \int_{0}^{y_{x, a, k}} 4 \sum_{j=1}^{n} \frac{1}{2^{j-1}} \frac{\left|x-b_{j}\right| t}{\left[\left(x-b_{j}\right)^{2}+a^{2}\right]^{2}} d t \\
& =2 \sum_{j=1}^{n} \frac{1}{2^{j-1}} \frac{\left|x-b_{j}\right| t^{2}}{\left[\left(x-b_{j}\right)^{2}+a^{2}\right]^{2}} \mid y_{x, a, k}^{y_{x, k}} \\
& =2 \sum_{j=1}^{n} \frac{1}{2^{j-1}} \frac{\left|x-b_{j}\right|}{\left[\left(x-b_{j}\right)^{2}+a^{2}\right]^{2}} \frac{\left[\left(x-b_{k}\right)^{2}+a^{2}\right]^{3 / 2}}{4} \\
& \leq \frac{1}{2} \sum_{j=1}^{n} \frac{1}{2^{j-1}} \frac{\left|x-b_{j}\right|}{\left[\left(x-b_{j}\right)^{2}+a^{2}\right]^{2}}\left[\left(x-b_{j}\right)^{2}+a^{2}\right]^{3 / 2} \\
& =\frac{1}{2} \sum_{j=1}^{n} \frac{1}{2^{j-1}} \frac{\left|x-b_{j}\right|}{\left[\left(x-b_{j}\right)^{2}+a^{2}\right]^{1 / 2}} \\
& \leq \frac{1}{2} \sum_{j=1}^{n} \frac{1}{2^{j-1}}
\end{aligned}
$$




$$
\begin{aligned}
& <\frac{1}{2} \sum_{j=1}^{\infty} \frac{1}{2^{j-1}} \\
& =1 .
\end{aligned}
$$

Set $\gamma_{x, a}(y)=F_{a}(x, y)$. Since $v_{a}(x, 0)=0$ and $\cos (1)>1 / 2$, combining (6) and (12), we get

$$
\begin{aligned}
\left\langle\gamma_{x, a}^{\prime}(y), \gamma_{x, a}^{\prime}(0)\right\rangle & =\cosh v_{a}(x, y) \cos \left(u_{a}(x, y)-u_{a}(x, 0)\right) \\
& >\cosh v_{a}(x, y) / 2
\end{aligned}
$$

where $\gamma_{x, a}^{\prime}(y)=\partial_{y} F_{a}(x, y)$. By (13), the angle between $\gamma_{x, a}^{\prime}(y)$ and $\gamma_{x, a}^{\prime}(0)$ is always less than $\pi / 2$, proving (ii) on $\Omega_{a, k}$, and hence on all of $\Omega_{a}$ since $k$ was arbitrary.

To prove (iii), note that, by (8) and (9), we have

$$
\partial_{y} v_{a}(x, y)=\sum_{j=1}^{n} \frac{1}{2^{j-1}} \frac{\left(x-b_{j}\right)^{2}+a^{2}-y^{2}}{\left[\left(x-b_{j}\right)^{2}+a^{2}-y^{2}\right]^{2}+4\left(x-b_{j}\right)^{2} y^{2}} .
$$

As before, fix $k, 1 \leq k \leq n$, and look on $\Omega_{a, k}$. Then, for all $j=1, \ldots, n$,

$$
\begin{aligned}
{\left[\left(x-b_{j}\right)^{2}+a^{2}-y^{2}\right]^{2}+4\left(x-b_{j}\right)^{2} y^{2} } & \leq\left[\left(x-b_{j}\right)^{2}+a^{2}+y^{2}\right]^{2} \\
& \leq\left[\left(x-b_{j}\right)^{2}+a^{2}+\frac{\left(x-b_{k}\right)^{2}+a^{2}}{4}\right]^{2} \\
& \leq\left[\left(x-b_{j}\right)^{2}+a^{2}+\frac{\left(x-b_{j}\right)^{2}+a^{2}}{4}\right]^{2} \\
& =\frac{25}{16}\left[\left(x-b_{j}\right)^{2}+a^{2}\right]^{2} . \\
\left(x-b_{j}\right)^{2}+a^{2}-y^{2} & \geq\left(x-b_{j}\right)^{2}+a^{2}-\frac{\left(x-b_{k}\right)^{2}+a^{2}}{4} \\
& \geq\left(x-b_{j}\right)^{2}+a^{2}-\frac{\left(x-b_{j}\right)^{2}+a^{2}}{4} \\
& =\frac{3}{4}\left[\left(x-b_{j}\right)^{2}+a^{2}\right] .
\end{aligned}
$$

So, we have

$$
\begin{aligned}
\partial_{y} v_{a}(x, y) & \geq \frac{12}{25} \sum_{j=1}^{n} \frac{1}{2^{j-1}} \frac{1}{\left(x-b_{j}\right)^{2}+a^{2}} \\
& >\frac{3}{8} \sum_{j=1}^{n} \frac{1}{2^{j-1}} \frac{1}{\left(x-b_{j}\right)^{2}+a^{2}} .
\end{aligned}
$$

Let $y_{x, a, k}=\frac{\left[\left(x-b_{k}\right)^{2}+a^{2}\right]^{3 / 4}}{2}$, as before. Since $v_{a}(x, 0)=0$, integrating (14) gives

$$
\begin{aligned}
\min _{\frac{y_{x, a, k}}{2} \leq|y| \leq y_{x, a, k}}\left|v_{a}(x, y)\right| & =\min _{\frac{y_{x, a, k}}{2} \leq|y| \leq y_{x, a, k}}\left|\int_{0}^{y} \partial_{y} v_{a}(x, t) d t\right| \\
& >\frac{3}{8} \int_{0}^{\frac{y_{x, a, k}}{2}} \sum_{j=1}^{n} \frac{1}{2^{j-1}} \frac{1}{\left(x-b_{j}\right)^{2}+a^{2}} d t \\
& \geq \frac{3}{8} \int_{0}^{\frac{y_{x, a, k}}{2}} \frac{1}{2^{k-1}} \frac{1}{\left(x-b_{k}\right)^{2}+a^{2}} d t
\end{aligned}
$$




$$
\begin{aligned}
& =\frac{3}{8} \frac{1}{2^{k-1}} \frac{1}{\left(x-b_{k}\right)^{2}+a^{2}} \frac{\left[\left(x-b_{k}\right)^{2}+a^{2}\right]^{3 / 4}}{4} \\
& =\frac{3}{32} \frac{1}{2^{k-1}}\left[\left(x-b_{k}\right)^{2}+a^{2}\right]^{-1 / 4} \\
& >\frac{\left[\left(x-b_{k}\right)^{2}+a^{2}\right]^{-1 / 4}}{11 \cdot 2^{n-1}} .
\end{aligned}
$$

Now, integrating (13) and using (15), we obtain

$$
\left\langle\gamma_{x, a}\left(y_{x, a, k}\right)-\gamma_{x, a}(0), \gamma_{x, a}^{\prime}(0)\right\rangle>\frac{\left[\left(x-b_{k}\right)^{2}+a^{2}\right]^{3 / 4}}{16} e^{\left[\left(x-b_{k}\right)^{2}+a^{2}\right]^{-1 / 4} / 11 \cdot 2^{n-1}}
$$

Since $\lim _{s \rightarrow 0} s^{3} e^{s^{-1} / 11 \cdot 2^{n-1}}=\infty$, (16) and its analog for $\gamma_{x, a}\left(-y_{x, a, k}\right)$ give an $r_{k}>0$ for which (iii) holds (with $r_{k}$ in place of $r_{0}$ ) on $\Omega_{a, k}$. This proves (iii) on all of $\Omega_{a}$, with $r_{0}=\min _{k} r_{k}$.

Corollary 6. Let $r_{0}$ be given by part (iii) of Lemma 5 , Then,

(a) $F_{a}$ is an embedding.

(b) $F_{a}(t, 0)=(0,0, t)$ for $|t|<1 / 2$.

(c) $\left\{0<x_{1}^{2}+x_{2}^{2}<r_{0}^{2}\right\} \cap F_{a}\left(\Omega_{a}\right)=\widetilde{\Sigma}_{1, a} \cup \widetilde{\Sigma}_{2, a}$ for multi-valued graphs $\widetilde{\Sigma}_{1, a}, \widetilde{\Sigma}_{2, a}$ over $D_{r_{0}} \backslash\{0\}$.

Proof. Same as [1, Cor. 1].

Proof of Theorem 2, By scaling, it suffices to find a sequence $\Sigma_{i} \subset B_{R}$ for some $R>$ 0 . By Corollary [6, there exist minimal embeddings $F_{a}: \Omega_{a} \rightarrow \mathbb{R}^{3}$ with $F_{a}(t, 0)=$ $(0,0, t)$ for $|t|<1 / 2$, so (iii) holds for any $R \leq r_{0}$. Set $R=\min \left\{r_{0} / 2,1 / 4\right\}$, and $\Sigma_{i}=B_{R} \cap F_{a_{i}}\left(\Omega_{a_{i}}\right)$, where the sequence $a_{i}$ is to be determined.

For each $j=1, \ldots, n$, by equation (10), we have $\left|K_{a}\right|\left(b_{j}\right) \rightarrow \infty$ as $a \rightarrow 0$, proving (i).

Also by (10), for each $j=1, \ldots, n$ and all $\delta>0$,

$$
\sup _{a} \sup _{\left\{\left|x-b_{j}\right| \geq \delta\right\} \cap \Omega_{a}}\left|K_{a}\right|<\infty
$$

for all $x \notin\left\{b_{1}, \ldots, b_{n}\right\}$. Combined with (iii) and Heinz's curvature estimate for minimal graphs (see, for example, [4, 11.7]), this proves (ii).

By Lemma 4, we can choose $a_{i} \rightarrow 0$ so that the $F_{a_{i}}$ converge uniformly in $C^{2}$ on compact subsets to $F_{0}: \Omega_{0} \rightarrow \mathbb{R}^{3}$. So, by Lemma 5 we obtain (iv)(a) and the decomposition $\Sigma^{k} \backslash\left\{x_{3}-\right.$ axis $\}=\Sigma_{1}^{k} \cup \Sigma_{2}^{k}$ for multi-valued graphs $\Sigma_{j}^{k}$, where $j=1,2$ and $k=1, \ldots, n+1$. To obtain (iv)(b) and the remainder of (iv)(c), we must show that each graph $\Sigma_{j}^{k}$ is $\infty$-valued, as this would imply the spiraling which we seek. By (iii) and (6), the level sets $\left\{x_{3}=x\right\} \cap \Sigma_{j}^{k}$ are graphs over the line in the direction

$$
\lim _{a \rightarrow 0}\left(\sin u_{a}(x, 0),-\cos u_{a}(x, 0), 0\right) .
$$

Since, for all $j=1, \ldots, n$ and all $t$ sufficiently close to $b_{j}$,

$$
\lim _{a \rightarrow 0}\left|u_{a}\left(t-b_{j}, 0\right)-u_{a}\left(2\left(t-b_{j}\right), 0\right)\right|=\frac{1}{2\left(t-b_{j}\right)},
$$

we see that, for $t$ sufficiently close to $b_{j},\left\{t-b_{j}<\left|x_{3}\right|<2\left(t-b_{j}\right)\right\} \cap \Sigma_{j}^{k}$ contains an embedded $N_{t}$-valued graph, where $N_{t} \approx 1 / 4 \pi\left(t-b_{j}\right) \rightarrow \infty$ as $t \rightarrow b_{j}$. This proves that each $\Sigma_{j}^{k}$ spirals the way we claim, completing the proof of (iv). 


\section{REFERENCES}

[1] T.H. Colding and W.P. Minicozzi II, Embedded minimal disks: proper versus nonproperglobal versus local, Trans. Amer. Math. Soc., 356, (2003), 283-289. MR2020033(2004k:53011)

[2] T.H. Colding and W.P. Minicozzi II, The space of embedded minimal surfaces of fixed genus in a 3-manifold IV; Locally simply connected, preprint, math.AP/0210119.

[3] W. Meeks and M. Weber, in preparation.

[4] R. Osserman, A survey of minimal surfaces, Dover, 2nd ed., (1986). MR0852409 (87j:53012) Department of Mathematics, Hylan Building, University of Rochester, Rochester, NEW York 14627

E-mail address: bdean@math.rochester.edu 